\title{
USER INVOLVEMENT IN ESTABLISHING A NEW YOUNG
} PEOPLE'S HOSPICE

Ray Kipling, Vicky Amos, Julia Goldsmith Naomi House, Winchester, UK

10.1136/bmjspcare-2011-000100.8

We propose that this presentation will involve life limited young people who have shaped a new hospice service for teenagers and young adults.

Transition from children's to adult services is much discussed, little understood and poorly supported. It poses a set of unique challenges for life limited young people.

Prime among these is the sharp discontinuity, described by many as like "falling off a cliff". The aim of the consultations was to shape a new service was to provide stability and continuity while facilitating transition across external health and social services.

By engaging user groups in a series of semi structured discussions, their needs were better understood and a new hospice service was established to support them. Each themed discussion had a facilitator. The format was kept loose to allow subjects to emerge that were important to the young people. In addition to focus groups of communicative users, carers were consulted on behalf of non-communicators, and there was a joint carer/young person session.

The results were that information was gathered on both physical and emotional needs. Three themes emerged; independence, dignity and privacy. In some areas, the young people's views were at odds with other stakeholders, such as parents/ cares and care professionals. It became apparent that young people's aspirations are often drowned out by carers and professionals.

Because the new hospice was founded on user involvement, it now provides a safe space for honest and open discussions. As trust is built, more profound subjects, such as relationships with peers and parents, advance care plans, death of friends and anxiety about sharing bad news, are being brought out. The best source of information for shaping young people's service is the young people themselves. Developing best practice, based on both their hopes and needs is a rewarding journey for all concerned 